\title{
The Effectiveness of Home Based Nursing Care in Treatment of Hospital Acquired Pressure Ulcer (HAPU) in Participants Seeking Home Health Services: An Exploratory Study
}

\author{
Punitha Singh ${ }^{1}$, Deepti Damodaran ${ }^{1}$, Gaurav Thukral ${ }^{1} \&$ Joseph P. C. ${ }^{1}$ \\ ${ }^{1}$ Health Care at Home India Pvt. Ltd, Noida, India \\ Correspondence: Dr. Gaurav Thukral, Chielf Opreating Officer (COO), Health Care at Home India Pvt. Ltd, \\ Noida, D-8, 1st Floor, Sector-3, Noida, Uttar Pradesh, 201301, India. Tel: 1800-102-4224.
}

Received: May 14, 2019

Accepted: May 31, 2019

Online Published: June 4, 2019

doi:10.20849/ijsn.v4i2.589

URL: https://doi.org/10.20849/ijsn.v4i2.589

\begin{abstract}
Hospital-Acquired Pressure Ulcers (HAPU) are serious clinical complications that can lead to increased length of stay, pain, infection, and, potentially, death. Unfortunately, a large proportion of home care clients received from hospital present with pressure ulcer that varies from stage 1 to stage 4 . Home care nurses have an opportunity to manipulate favorably certain environmental factors that can prevent pressure ulcers from newly developing or to manage effective treatment plans for any stages ulcers which were present while onboarding.

Objective: The objective of this study was to determine the prevalence, duration and outcome in terms of healing; worsening of pressure ulcers and the ones whose condition remained the same seeking home health services. The study also describes the home care nursing interventions done to minimize the risk of worsening of pressure ulcer.
\end{abstract}

Keywords: pressure ulcer, pain, infection, Hospital Acquired Pressure Ulcer (HAPU), wound healing, interventional study

\section{Introduction}

Pressure ulcer is a common, serious, and significant healthcare occurrence among hospitalized clients. A pressure ulcer, also called as bedsore, is an injury to the skin or underlying tissue caused by constant pressure, friction and moisture. These ulcers often occur when clients have limited mobility can't change position in bed on their own.

The National Pressure Ulcer Advisory Panel (NPUAP) (Note 1) defines a pressure ulcer as "localized injury to the skin and/or underlying tissue usually over a bony prominence, as a result of pressure, or pressure in combination with shear and/or friction".

Pressure ulcers develop when capillaries supplying the skin and subcutaneous tissues are compressed enough to impede perfusion, leading ultimately to tissue necrosis. When pressure ulcers occur, they must be treated quickly, or they can damage the skin and muscles leading to slow recovery and causes infection and other problems. This complication causes pain, disfigurement, slow recovery from co-morbid conditions, and interferes with activities of daily living. Clients are at risk for pressure ulcers when they can't move easily, are confined to bed, advanced age leading to decreased circulation or fragile skin. Changes in cognitive or bowel and bladder function, along with insufficient intake of nutrients and liqids, can also contribute to the development of pressure ulcers.

Pressure ulcer prevention is an important nursing role and frequently used benchmark for quality care. Enhancing and reinventing the role of Home Health Care Nurses are critical in promoting patient safety and overall patient outcomes. The challenge is more difficult when there is nursing staff turnover and shortages. Studies have suggested that pressure ulcer development can be directly affected by the number of registered nurses and time spent at the bedside.

A Cohort Study was carried out to estimate the patterns of care and annual levels of healthcare resource use attributable to managing pressure ulcers (PUs) in clinical practice in the community by the UK's National Health Service (NHS), and the associated costs of patient management. Results suggested that $50 \%$ of all the PUs healed within 12 months from initial presentation, but this varied between $100 \%$ for category 1 ulcers and $21 \%$ for category 4 ulcers. The mean time to healing ranged from 1.0 month for a category 1 ulcer to 8 months for a 
category $3 / 4$ ulcer and 10 months for an unstageable ulcer. Clients were predominantly managed in the community by nurses with minimal clinical involvement of specialist clinicians (Note 2).

An experimental study to identify the effectiveness of preventive interventions on prevalence of Hospital Acquired Pressure Ulcer (HAPU) suggested the multidisciplinary approach was effective in decreasing the prevalence of HAPUs. Skin care management which was a significant predictor of PUs should alert nurses to the cost effectiveness of this intervention. The study also suggested that two factors significantly predicted the development of a HAPU; Skin care and Braden score (Note 3). The Braden Scale is designed for use with adults and consists of 6 subscales: sensory perception, moisture, activity, mobility, nutrition, and friction and shear. The scores on this scale range from 6 (high risk) to 23 (low risk), with 18 being the cut score for onset of pressure ulcer risk (Note 4).

Previous studies on pressure ulcer prevention using questionnaire surveys have targeted physicians and nurses working in hospitals. However, few have administered surveys to nurses in home care services.

Hence, this study was conducted to identify adaptation of Pressure Ulcer Prevention Guidelines for use in Home Health Care setting. The purpose of this study was to delineate interventions and activities used to prevent PU in home health care. Nurses used an array of interventions, including those recommended by clinical practice guidelines and physician instructions to prevent PU in home health clients. Findings provide insight into guideline adaptation in home health care setting and suggest that PU prevention in the home health care setting is more complex than that in hospitals and nursing homes requires significant skills in communication and collaboration to prevent the occurrence of pressure ulcer and reduce the risk factors. The ultimate goal of this study is to device evidence-based prevention interventions and training of staff in the reduction of overall HAPUs to promote patient safety and optimum clinical outcomes.

\section{Method}

This Exploratory Study monitored and reviewed the treatment plan of clients with pressure ulcer to identify the following parameters Figure 1.

- Number of clients with HAPU received under Home Care Nursing Package

- Number of clients whose pressure ulcer healed

- Number of clients whose pressure ulcer condition worsened

- Number of clients whose pressure ulcer condition improved

- Number of clients whose pressure ulcer stage remained in same condition

- Number of clients who developed new bed pressure ulcer

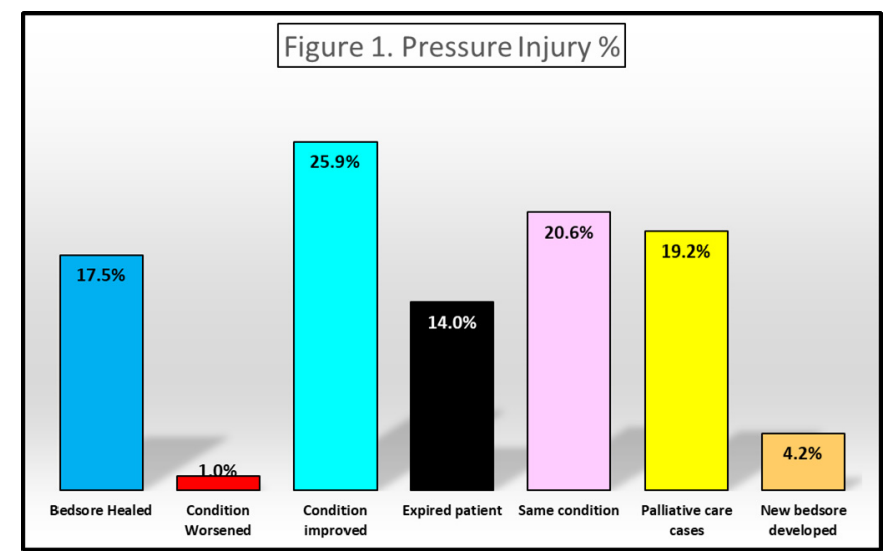

Figure 1. Inclusion parameters for pressure ulcer identification

Monthly incident reports of all pressure ulcers identified were reported by nursing staff through the Patient Care System (PCS). The data of one year from April 2018 to March 2019 were collected and verified by the Clinical Excellence and Quality Team (CET). Although few new home acquired stage 1 pressure ulcers were also reported, our priority was in reducing and preventing worsening of Hospital Acquired Pressure Ulcers.

March 2019 data showed a total of 21 clients with Pressure Ulcer. Of these 20 were Hospital Acquired Pressure 
Ulcer (HAPU) and one was home acquired. Of the 20 HAPU, four clients displayed same condition of pressure ulcer due to cultural relativeness of patient in regard to nutrition, comorbidities like Diabetes and other underlying disease condition that impaired healing, age related factors that slowed down healing process and refusal to advanced or change in treatment especially dressings.

\subsection{Identify Subsections}

Our main objective was to improve the condition of clients received at home from hospital with pressure ulcers. Weekly and monthly review meetings were organized between nursing team, physicians, clinical excellence and quality team and a plan of action was put into force.

The five main areas targeted were:

- Improving staff education and knowledge of pressure ulcer prevention

- Accurate and timely risk assessment of the patient using Braden Score

- Frequent patient offloading/relieving pressure

- $\quad$ Skin moisture management.

- Improving nutritional status

In relation to improving staff education and knowledge of pressure ulcer prevention, the training team along with the clinical excellence team ensured all pressure ulcer prevention guidelines and policies were reviewed and updated and staff was educated to follow them. Pressure ulcer risk assessment, prevention and management was included as part of the induction and refresher classes for nurses to ensure better understanding. Overall these lectures were very well attended by the home care ICU staff and evaluation forms and feedback demonstrated a positive reinforcement of prevention and identifying at risk clients.

In relation to risk assessment, it is also policy of the organization to carry out an initial skin risk assessment upon onboarding the patient and also in every shift during the provision of home care. Nursing staff were encouraged to assess the risk of clients within this time frame and complete a skin assessment as part of daily assessment in the Patient Care System (PCS) every time they repositioned the patient. A validated risk assessment scale called the Braden scale4 which was used to identify clients at risk of developing pressure ulcers. The lower the score the higher the risk and any patient with a score of 18 or below are deemed at risk. The clinical excellence team monitored the risk assessment and modified patient care plan accordingly including preventive measures. It was decided to reposition, turn and off load clients every two hours instead of every three hours. Pressure relieving matresses were used as part of preventive measures

Another area examined was managing moisture via incontinence care. Patient was kept dry and skin care done everytime patient passed stool or urine. It had also been noted in the data collected that many of the clients who developed hospital acquired pressure ulcers in the ICU department of hospital due to faecal incontinence. This was taken care of at home care environment and it was made sure that meticulous skin care is done whenever patient had incontinence and also skin was assessed for risk in every shift.

Adequate nutrition was ensured with the support of the nurtitonist and the nurses were made aware of the effect of nutruional support in healing of pressure ulcers. This was monitored on a weekly basis whenever multi-disciplinary team meets centrally to discuss patient progress.

\subsection{Participant (Subject) Characteristics}

The study was conducted among clients undergoing home care nursing services who developed pressure ulcer during hospital stay. They were monitored after their discharge from hospital. This also included few clients who developed new pressure ulcer during the study.

\subsection{Sampling Procedures}

Simple Random Sampling was done to identify the subjects for the study. The study was conducted among the clients with Hospital Acquired Pressure Ulcer (HAPU) under Home Care Nursing services.

\subsubsection{Sample Size, Power, and Precision}

The sample consisted of clients with Hospital Acquired Pressure Ulcer (HAPU) under Home Care Nursing services. This included an average of 24 clients in a month from April 2018 to March 2019, having various stages of pressure ulcer developed during their hospital stay.

\subsubsection{Measures and Covariates}

The pressure ulcer prevention protocol consisted of preventive interventions stratified on risk level assessment 
using Braden's scale, with implementation of support services including skin care and turning/repositioning the patient/ ensuring mainatance of moisture/ nutrition and relief of pressure. One year PU data from April 2018 to March 2019 was considered. Figure 2 shows HAPU prevalence over a 12-month period, which was divided into 4 quarters (Q1 to Q4). An average of 24 clients was part of the study sample; who evidenced HAPU. The trend in the average number of clients with pressure ulcer was highest in Q2 (29.6) and lowest in Q4 (19).

The Braden's scale for risk assessment was used for data collection in every shift and entered in the Patient Care System (PCS) and analysis done on monthly basis. The Health Care at Home (HCAH) Nursing Manual was used as reference guide in training the staff nurses on prevention and management of pressure ulcer (Note 5).

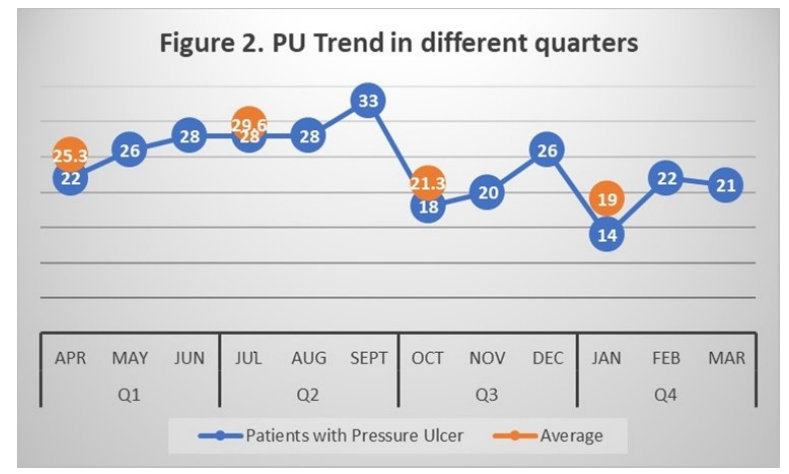

Figure 2. PU Trend in different quarters

\subsubsection{Research Design}

Descriptive study design was used to collect data from clients with pressure ulcer using pressure ulcer risk assessment tool i.e. the Braden's scale on daily basis. The data was collected over a period of one year from April 2018 to March 2019 for analysis and interpretation done.

\subsubsection{Experimental Manipulations or Interventions}

The five main areas targeted were to improve staff education and knowledge of pressure ulcer prevention, accurate and timely risk assessment of the patient using Braden Score, frequent patient offloading/relieving pressure, skin moisture management and improving nutritional status

\section{Results}

The data collected was analysed and summarized and the following observation was made after preventive interventions were implemented. Figure 3 suggested that out of the 24 clients reported to have pressure ulcer, $17 \%$ of clients healed completely among which, Degree I and Degree II pressure ulcer healed completely over a period of 15 to 20 days and clients with III- and IV-degree pressure ulcer showed subsequent improvement in the stage of pressure ulcer. $25.9 \%$ showed improvement in the degree of pressure ulcer, $20.6 \%$ showed no signs of improvement or deterioration, none of them showed worsening in the wound condition and only one developed a new pressure ulcer due to age leading to fragile skin and some cultural consideration in taking the diet suggested by physician.

Further it was also observed that there were about 20 clients with Degree II pressure ulcer in the month of September which subsequently decreased to 13 clients by March, which suggests reduction in the number of clients with Degree II pressure ulcer which could be due to early identification and adherence to pressure ulcer prevention and management guidelines. 


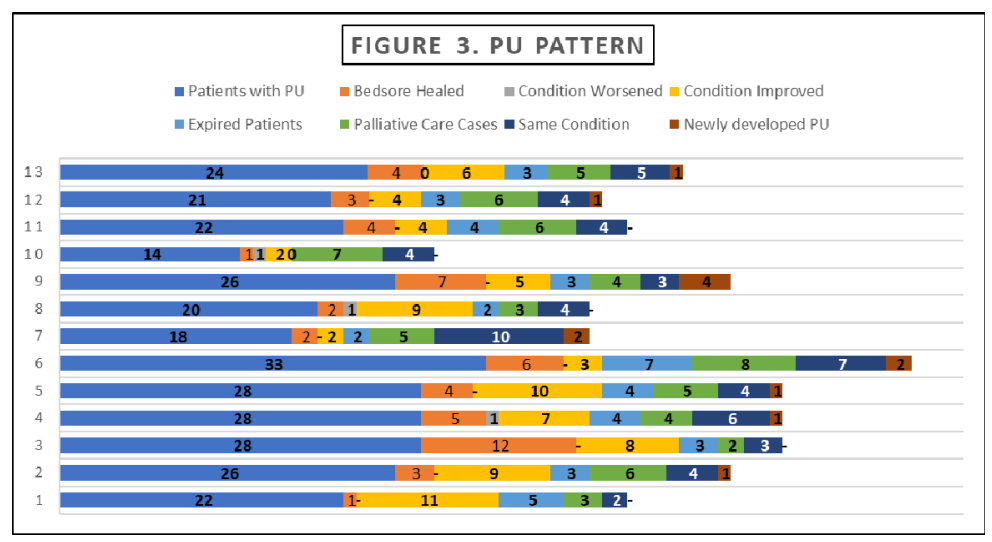

Figure 3. PU pattern

\section{Discussion}

A hospital-acquired pressure injury (HAPI; formerly known as a pressure ulcer) is a localized injury to the skin and/or underlying tissue during an inpatient hospital stay (Note 6). The result of pressure, shear, or both, HAPI development is additionally associated with other factors (e.g., advanced age, immobility, perfusion, nutritional status, hematological measures, illness severity, and presence of diabetes. HAPU prevalence over a 12-month period, which was divided into 4 quarters (Q1 to Q4). An average of 24 clients was part of the study sample; who evidenced HAPU. The mean age of the clients was from 50 to 80 years. Pressure ulcer stage and location were tracked. Pressure ulcer was identified at right and left iliac crest, coccyx, scarum and heel of foot. Pressure ulcer stages ranged from a maximum of 13 cases of stage II pressure ulcer, 4 cases of stage I pressure ulcer, 3 cases of stage III pressure ulcer and 2 cases of stage IV pressure ulcer. No case of unstageable or suspected deep tissue injury pressure was found. Further it was also observed that there were about 20 clients with Degree II pressure ulcer in the month of September which subsequently decreased to 13 clients by March, which suggests reduction in the number of clients with Degree II pressure ulcer.

The trend in the average number of clients with pressure ulcer was highest in Q2 (29.6) and lowest in Q4 (19). The overall trends in the HAPU prevalence rates showed a consistent downward trend from 29.6 to 19 after the implementation of preventive measures including teaching and training of staff on prevention and management of pressure ulcer, correct and timely risk assessment of the patient, frequent patient offloading/relieving pressure, and moisture management.

The majority of our clients had a high-risk score of 10 to 14 as observed in the Braden's Scale. The data suggests that critically ill clients are at increased risk of developing pressure ulcer because of the existing disease condition along with impared mobility, hemodynamic instability, excessive moisture, co-morbidities like Diabetes Mellitus and Hypertension and suboptimal nutrition.

Recently the National Pressure Ulcer Advisory Panel released a statement that not all PUs is avoidable. The panel of experts agreed that certain situations create conditions such as hemodynamic instability seen in clients in critical care units, may influence turning or reposition, and lead to unavoidable Pus (Note 7). This statement, however, does not mean that PU assessment risk and prevention strategies should be minimized. Rather, the critically ill patient is at greatest risk of PU; thus, critical care nurses need to be vitally involved in the prevention of PU through ongoing skin and PU risk assessment and initiating interventions to minimize clients' risk for developing a HAPU.

We found that peer-to-peer discussions during bedside rounds in form of audits and daily Critinext round in terms of video calls were perceived to be more effective and meaningful to critical care nurses than were typical educational strategies of online education modules and classroom staff in-service education. Nursing rounds provide a forum to facilitate collaborative problem identification, clinical decision making, and incorporation of evidence at the point of care to promote individualized patient-centered evidence-based nursing practice. Video rounds by Critinext team and bed side audits allowed sharing of clinical information and PU-prevention education that was seen as immediately meaningful to critical nurses. The result of this study is limited to the cultural beliefs of patient and family members in terms of diet modification and refusal to treatment for advanced or change in treatment. 


\section{Conclusion}

Hospital-Acquired Pressure Ulcer (HAPU) is not only a major concern in hospital and continues to be an alarming issue in today's healthcare systems. Often preventable, it is a significant nursing role and a frequently used benchmark for quality care. And even though pressure ulcer prevention is a fundamental intervention that is not new, is inexpensive, and affects patient safety and quality outcomes, HAPU prevalence continues to rise.

Enhancing and reinventing the role of Home Health Care Nurses are critical in promoting patient safety and overall patient outcomes. The challenge is more difficult when there is nursing staff turnover and shortages. Therefore, the study was conducted to identify adaptation of Pressure Ulcer Prevention Guidelines for use in Home Health Care setting to delineate interventions and activities used to prevent PU in home health care. Certified nurses used an array of interventions, including those recommended by clinical practice guidelines, to prevent PU in home health patients.

The major areas targeted were, improving staff education and knowledge of pressure ulcer prevention, accurate and timely risk assessment of the patient using Braden Score, frequent patient offloading/relieving pressure, skin moisture management, improving nutritional status.

Findings provide insight into guideline adaptation in home health care and suggest that PU prevention in the home health care setting is more complex than that in hospitals and nursing homes and requires significant skills in communication and collaboration. It was concluded that home health care nursing played a major role in decreasing the worsening of HAPU by giving individualized care to the patient and taking care of the smallest details in preventing the worsening and development of new Pressure Ulcer. Further study may be conducted in this area by taking a larger sample for generalization and also conducting a longitudinal study to see the response of patient to a specific intervention over a longer period of time.

\section{Limitations of the study}

Involving key stakeholders and a multi-disciplinary team to identify and improve patient care can be difficult to coordinate, but it can lead to a more successful outcome. Some healthcare professionals have a more positive attitude towards a team approach than others and are more accommodating. Unfortunately, the study interventions were limited to the fact that patient's next of kin showed some reluctance to adapt the nutritional modifications suggested by clinical team for the patient due to cultural beliefs that delayed wound healing process. Also, few patient's family exhibited refusal to treatment in terms of adapting advanced treatment options like silicone foams in case of advanced stage of pressure ulcer.

The study was limited for a period of one year, it may be considered to conduct the study over a long period of time approximately 36 months which is divided into 6 quarters, to have effective results.

\section{Acknowledgements}

We thank all our nursing staff, clinical excellence team, quality and training team for their contribution and mentorship with this quality improvement project.

\section{References}

Aitken, L. M., Burmeister, E., Clayton, S., et al. (2011). The impact of nursing rounds on the practice environment and nurse satisfaction in intensive care: pre-test post-test comparative study. Int J Nurs Stud., 48, 918-925. https://doi.org/10.1016/j.ijnurstu.2010.10.004

Bergquist-Beringer, S., \& Daley, C. M. (2011, March/April). Adapting Pressure Ulcer Prevention for Use in Home Health Care; Bergquist-Beringer, Sandra Daley. Christine Makosky Journal of Wound, Ostomy and Continence Nursing, 38(2), 145-154. https://doi.org/10.1097/WON.0b013e31820ad115

Frumenti, J., \& Kurtz, A. (2014). Addressing Hospital-Acquired Pressure Ulcers: Patient Care Managers Enhancing Outcomes at the Point of Service. JONA: The Journal of Nursing Administration, 44(1), 30-36. https://doi.org/10.1097/NNA.0000000000000018

Guest, J. F., Fuller, G. W., Vowden, P., \& Vowden, K. R. (2018). Cohort study evaluating pressure ulcer management in clinical practice in the UK following initial presentation in the community: costs and outcomes. BMJ Open, 8(7), e021769. https://doi.org/10.1136/bmjopen-2018-021769

Howard, D. C. (2019). Improving Nursing Staffing Methodology and Nursing Sensitive Outcomes with the Addition of a Patient Centered Acuity Measure. Doctor of Nursing Practice Projects, George Washington University.

Institute for Healthcare Improvement. (n. d.). Pressure Ulcer Prevention. Retrieved April 24, 2011, from 
http://www.ihi.org

Kottner, J., Cuddiganc, J., Carvilled, K., Balzere, K., Berlowitzfg, D., Lawh, S., ...Haeslertuv, E. (2019). Prevention and treatment of pressure ulcers/injuries: The protocol for the second update of the international Clinical Practice Guideline 2019. Journal of Tissue Viability, 28(2), 51-58. https://doi.org/10.1016/j.jtv.2019.01.001

Loewenthal, A. (2016). Reducing the Incidence of Hospital-Acquired Pressure Ulcers by Enhancing the Role of UnitBased Skin Champions. Master's Projects and Capstones, The University of San Francisco.

\section{Links}

https://www.ncbi.nlm.nih.gov/pmc/articles/PMC6067374/

https://www.ucsfhealth.org/about/pressure-ulcers/

https://journals.lww.com/jonajournal/Abstract/2014/01000/Addressing_Hospital_Acquired_Pressure_Ulcers_.9.a $\operatorname{spx}$

https://www.researchgate.net/publication/236080431_The_Value_of_Reducing_Hospital-Acquired_Pressure_Ul cer_Prevalence

https://www.sciencedirect.com/science/article/pii/S0965206X18301190

https://hsrc.himmelfarb.gwu.edu/son_dnp/43/

\section{Notes}

Note 1. National Pressure Ulcer Advisory Panel. Not all pressure ulcers are avoidable. http://www.npuap.org/A_UA\%20Press\%20Release.pdf. Accessed September 21, 2011.

Note 2. The Effectiveness of a Pressure Ulcer Intervention Program on the Prevalence of Hospital Acquired Pressure Ulcers: Controlled Before and After Study. Zeinab Mallaha, Nada Nassara, Lina Kurdahi Badrb.

Note 3. Johnson-Pawlson J, Infeld DL. Nurse staffing and quality of care in nursing facilities. J Gerontol Nurs. 1996, 22(8), 36-45.

Note 4. Braden BI, Bergestrom N. Clinical utility of the Braden Scale for Predicting Pressure Sore Risk. Decubitus. 1989, 2, 44-51.

Note 5. Health Care at Home, L1-06, Nursing Manual, V1, Procedure 7.1, Back Care.

Note 6. Hospital-Acquired Pressure Injury; Risk-Adjusted Comparisons in an Integrated Healthcare Delivery System.

Note 7. June Rondinelli, PhD, RN, CNS, Stephen Zuniga, PhD, Patricia Kipnis, PhD, Lina Najib Kawar, PhD, RN, CNS, Vincent Liu, MD, MS, and Gabriel J. Escobar, MD.

\section{Copyrights}

Copyright for this article is retained by the author(s), with first publication rights granted to the journal.

This is an open-access article distributed under the terms and conditions of the Creative Commons Attribution license (http://creativecommons.org/licenses/by/4.0/). 